\title{
Chironomidés (Diptera) du Massif Central et des basses régions avoisinantes
}

\author{
H. Laville1 \\ B. Serra-Tosio²
}

Mots clés : Dipterà, Chironomidae, faunistique, massif montagneux, plaine.

Le catalogue actualisé des Chironomidés du Massif Central français et des basses régions avoisinantes est établi à partir de travaux récents et même parfois inédits.

261 espèces, dont 60 nouvelles pour la Zone 8 (Massifs montagneux de moyenne altitude) de la Limnofauna Europaea. sont recensées. Le total des espèces de Chironomidés connus du Massif Central et des Pyrénées s'élève actuellement à 370 .

Chironomids (Diptera) of the Massif Central and neighbouring lowland regions.

Keywords : Diptera, Chironomidae, faunistic, mountain, lowland.

A catalogue of chironomids from the French Massif Central and neighbouring lowland regions was produced partially from recent publications and also from unpublished records.

261 species were recorded, with 60 being new records for Zone 8 (mountain regions at mean altitude) in the Limnotauna Europaea. The total number of chironomid species recorded for the Massif Central and Pyrenees has now increased to 370 .

Dans le cadre d'une mise à jour de la Faune des Chironomidés de France, il nous a semblé approprié d'effectuer une série d'inventaires limités aux principales zones biogéographiques de la Limnotauna Europaea qui intéressent le territoire : les Pyrénées $\left(Z_{2}\right)$, la Corse $\left(Z_{3}\right)$, les Alpes $\left(Z_{4}\right)$, les massifs de moyenne altitude $\left(Z_{8}\right)$, les plaines oxcidentales $\left(Z_{13}\right)$.

Le Massif Central fait partie des moyennes montagnes occidentales de l'Europe qui, avec les massifs des Vosges, de la Forêt Noire et du Jura, constituent la Zone 8 de la Limnofauna. Il représente la partie la plus méridionale et la plus haute de cette zone dont les altitudes maximales se situent respectivement à $1424 \mathrm{~m}$ (Vosges), $1493 \mathrm{~m}$ (Forêt Noire). $1718 \mathrm{~m}$ (Jura) et $1885 \mathrm{~m}$ (Massif Central). Les récoltes les plus intensives de Chironomidés dans le

1. Labora toire d'Hydrobiologie, UA.695 C.N.R.S., Université Paul Sabatier, 118 route de Narbonne, 31062 Toulouse Cédex, France.

2. Laboratoire de Zoologie. Université de Grenoble I, Domaine Universitaire, B.P. 68, 38042 Saint-Martin-d'Hères Cédex, France.
Massif Central ont été effectuées ces vingt dernières années et concernent essentiellement :

1. Les lacs de la région de Besse-en-Chandesse dans le Puy-de-Dôme entre $880 \mathrm{~m}$ et $1200 \mathrm{~m}$ (Verneaux 1968);

2. Les rivières du Sud-Ouest, affluents ou sousaffluents rive droite de la Garonne :

. Bassin du Lot : Rivières Lot entre $1295 \mathrm{~m}$ et $26 \mathrm{~m}$ et Truyère à $228 \mathrm{~m}$ (Laville 1979, 1981 : BrouquetLaglaire 1985) ;

. Bassin du Tarn : Rivière Aveyron de la source $(670 \mathrm{~m})$ à la confluence du Tarn $(80 \mathrm{~m})$ (ViaudChauvet 1981, Laville \& Viaud-Chauvet 1985); Rivière Tarn et son affluent l'Agout avec ses sousaffluents Dadou et Thoré entre $400 \mathrm{~m}$ et $74 \mathrm{~m}$ (ViaudChauvet 1981, Brouquet-Laglaire 1985);

. Bassin de la Dordogne : Rivière Dordogne de la source $(1320 \mathrm{~m})$ à la confluence avec la Gironde (90 m) (CEMAGREF 1981, Petitjean 1981).

Dans ces rivières du Sud-Ouest, seulement 19 stations, sur les 72 prospectées, sont situées au-dessus 
Tableau I. Inventaire 1987 des Chironomidés du Massif Central et des basses régions avoisinantes. Les nombres renvoient aux références bibliographiques :

romains : récoltes au-dessus et au-dessous de $500 \mathrm{~m}$;

soulignés : récoltes au-dessus de $500 \mathrm{~m}$;

italiques : récoltes au-dessous de $500 \mathrm{~m}$;

- : espèces nouvelles pour la zone 8 de la Limnofauna Europara 1978 ;

- : espèces seulement signalées au-dessous de $500 \mathrm{~m}$;

* : espèces non connues des pyrénées (Laville * vinçon 1986).

Tanypodinae (24 espèces)

Ablabesmyia longistyla Fitt. : (1) (4) (5) (21)

A. monilis (L.) : (3) (4) (20)

Apsectrotanypus trifascipennis (zett.) : (20)

* Arctopelopia griseipennis (v.d.w.) : (?4)

- Conchapelopia melanops (wied) = (1)

C. pallidula (Mg.) : (1) (4) (5) (20)

- C. viator (K.) : (2)

Macropelopia nebulosa (Mg.) : (1) (20) (21)

Natarsia punctata (Fabr.) : (1) (20)

Nilotanypus dubius (Mg.) : (1) (2) (4) (5) (7) (9) (21)

Paramerina divisa (Walk.) : (ㅁ) (21)

Procladius choreus (Mg.) : (4) (20)

* P. signatus (zett.) : (20)

Psectrotanypus varius (Fabr.) : (1) (20)

- Rheopelopia ornata (Mg.) : (1) (21)

* Tanypus punctipennis Mg. : (20) (21)

* Telopelopia fascigera (Vern.) = (6) (21)

Thienemannimyia carnea (Fabr.) : (4) (5)

Th. geijskesi (G.) : ()

* $\rightarrow$ Th. lentiginosa (Fries) : (?마 (?21)

$\rightarrow$ Th. northumbrica (Edw.) : (21)

* Th. vitellina (K.) : (1) (4)

Thissopelopia longimanus (St.) : (21)

Zavrelimyia hirtimanus (k.) : (21)

Buchonomyilnae (1 espèce)

* $\rightarrow$ Buchonomyia thienemanni fitt.: (1) (4, sub E.crassipes (Pz.)) (5) (21) 
Diamesinae (16 espèces)

Boneoheptagyia legeri (G.) (= Heptagyia punctulata G.) : (17) (19)

Diamesa aberrata Lundb. : (11) (14) (15)

D. bertrami Edw. : (11) (14) (15)

D. cinerella Mg.: (11) (14) (15) (22)

D. hamaticornis K.: (5) (13) (14) (15) (16) (20)

D. incallida (wallk.) : (14) (15)

D. insignipes K. : (10) (14) (15) (21)

D. Latitarsis G. : (2)

D. permacra (Walk.) : (14) (15)

D. tonsa (Hal.) (= thienemanni $\mathrm{K}$.$) : (2) (10) (11) (14) (15) (20) (?21) (22)$

D. zernyi Edw. : (10) (11) (14) (15)

Potthastia gaedii (Mg.) : (1) (4) (5) (9) (?12) (?14) (?15) (21)

Potthastia longimanus K. : (1) (2) (4) (5) (14) (15) (20) (21)

- Sympotthastia macrocera ser.-Tos. : (12) (14) (15)

- S. spinifera ser.-Tos. : (12) (14) (15)

- S. zaureli Pag. : (12) (14) (15)

Prodiamesinae ( 1 espèce)

Prodiamesa olivacea (Mg.) : (1) (3) (4) (5) (20) (21)

Orthocladilinae (111 espèces)

Brillia longifurca K. : (1) (2) (4) (5) (20) (21)

Brillia modesta (Mg.) : (1) (4) (5) (21)

* $\rightarrow$ Bryophaenocladius flexidens (Br.) : (5)

* B. muscicola (K.) : (5)

$\rightarrow$ B. subvernalis (Edw.) : (21)

* B. vernalis (G.) : (2) (4)

Camptocladius stercorarius (d. Geer) : (21)

$\rightarrow$ Cardiocladius capucinus (zett.) : (1) (2) (4) (5)

- c. fuscus K. : (1)

Chaetocladius perennis (Mg.) : (20) (21)

Corynoneura celtica Edw. : (?21)

* $\rightarrow \quad$ C. edwardsi Br. : (5)

- C. Lacustris Edw. : (4)

C. Lobata Edw. : (ㅁ) (21)

* Cricotopus albiforceps (K.) : (1) (2) (4) (5) (9) (21)

* $\rightarrow$ C. algarum $(\mathrm{K}):.(? 21)$ 


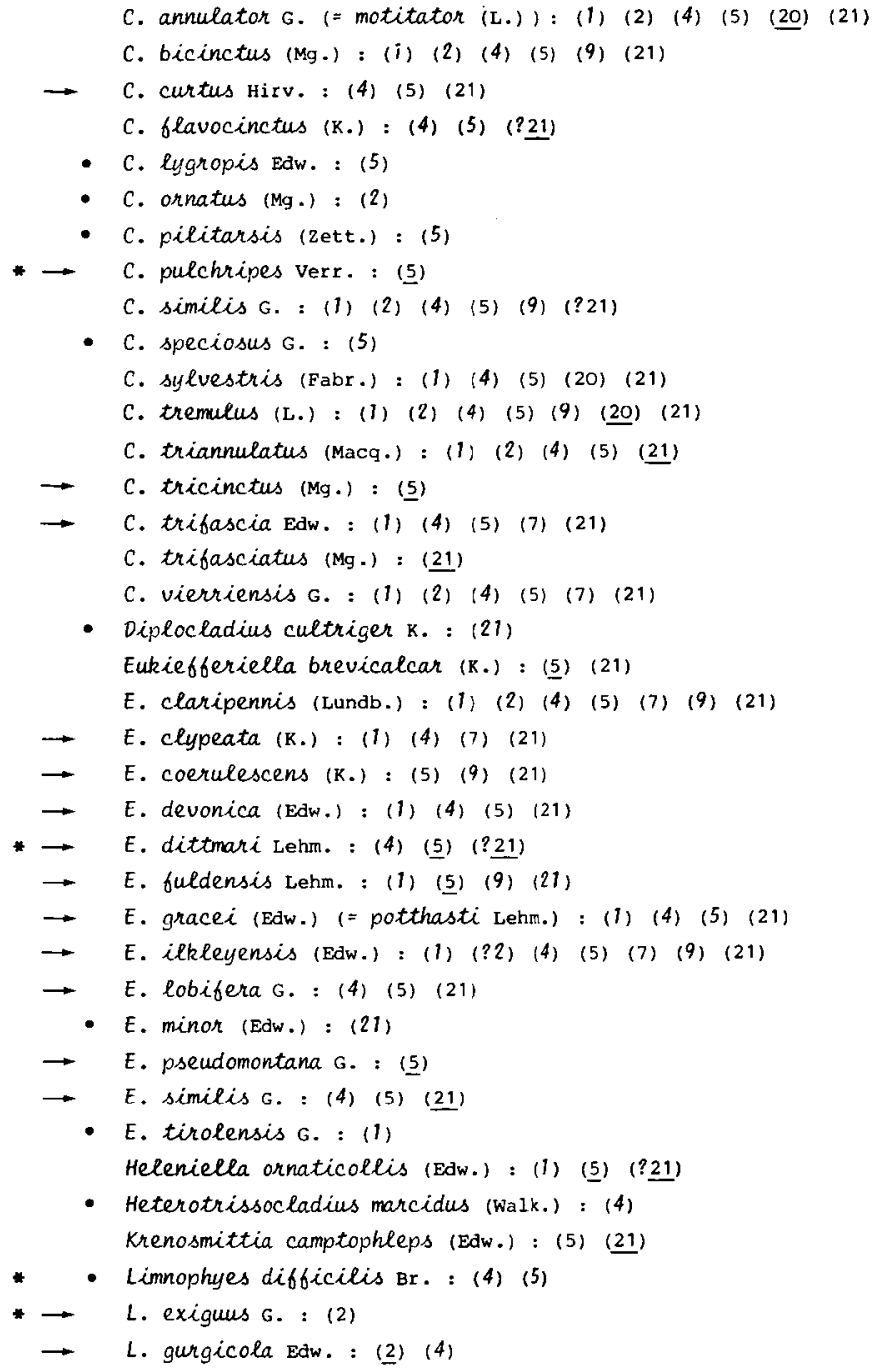

- C. speciosus G.: (5)

C. sylvestris (Fabr.) : (1) (4) (5) (20) (21)

c. tremulus (L.) : (1) (2) (4) (5) (9) (20) (21)

C. triannulatus (Macq.) : (1) (2) (4) (5) (21)

$\rightarrow$ C. tricinctus (Mg.) : (ㅁ)

$\rightarrow$ C. trifascia Edw. : (1) (4) (5) (7) (21)

C. trifasciatus (Mg.) : (21)

C. vierriensis G. : (1) (2) (4) (5) (7) (21)

- Diplocladius cultriger k. : (21)

Eukiefferiella brevicalcar (K.) : (5) (21)

E. claripennis (Lundb.) : (1) (2) (4) (5) (7) (9) (21)

$\rightarrow$ E. clypeata (K.) : (1) (4) (7) (21)

$\rightarrow$ E. coerulescens (K.) : (5) (9) (21)

$\rightarrow$ E. devonica (Edw.) : (1) (4) (5) (21)

* $\rightarrow$ E. dittmari Lehm. : (4) (5) (?21)

$\rightarrow$ E. fuldensis Lehm. : (1) (5) (9) (21)

- E. gracei (Edw.) (= potthasti Lehm.) : (1) (4) (5) (21)

$\rightarrow$ E. ilkleyensis (Edw.) : (1) (?2) (4) (5) (7) (9) (21)

$\rightarrow$ E. Lobifera G. : (4) (5) (21)

- E. minor (Edw.) : (21)

$\rightarrow \quad$ E. pseudomontana $G$. : (5)

- E. similis G. : (4) (5) (21)

- E. tirolensis G. : (1) Heleniella ornaticollis (Edw.) : (I) (5) (?21)

- Heterotrissocladius marcidus (walk.) : (4) Krenosmittia camptophleps (Edw.) : (5) (21)

* Limnophyes difbicilis Br. : (4) (5)

* $\rightarrow$ L. exiguus G. : (2)

$\rightarrow \quad$ L. gurgicola Edw. : (2) (4) 
$\rightarrow$ L. minimus $\mathrm{Mg}$. : (4) (21)

L. prolongatus K.: (4) (5) (19) (21)

- L. scapellatus Br. : (?21)

- L. truncorum G. : (4)

* Metriocnemus atriclavus $\mathrm{k}$. : (21)

M. Guscipes (Mg.) : (20) (21)

M. hygropetricus $\mathrm{K}$. : (ㅁ) (19) (20)

* $\rightarrow$ M. ursinus (Holm.) : (?21)

Nanocladius balticus (Pal.) : (1) (4) (21)

N. bicolor (zett.) : (1) (4) (5) (7) (9) (21)

$\rightarrow$ N. parvulus (K.) : (1) (4) (5) (9) (21)

N. rectinervis (K.) : (1) (2) (4) (5) (7) (9) (21)

$\rightarrow$ Orthocladius excavatus Br. : (1) (4) (5) (9) (21)

0 . Grigidus (zett.) : ( 5$)$ (20) (21)

o. fuscimanus (K.) (= bipunctellus auct.) : (1) (19, sub hygropetricus $\left.K_{.}\right)$

* 0. glabripennis (G.) : (2)

$\rightarrow \quad 0$ oblidens Br. : (1) (4) (?5) (21)

0 . obtexens $\mathrm{Br}$. : (4) (5) (?21)

o. rivicola K.: (1) (4) (5) (7) (9) (21)

O. rivulorum $\mathrm{K}$. : (4) (5) (7) (21)

0 . rubicundus (Mg.) : (2) (4) (20)

$\rightarrow$ 0. saxicola K. : (1) (?2) (4) (5) (7) (9) (21)

$\rightarrow \quad 0$. saxosus Tok. : (5)

0 . thienemanni $\mathrm{K}$. : (? 5 ) (20) (21)

Paracladius conversus (walk.) : (1) (21)

Paracricotopus niger (K.) : (1) (4) (5) (9) (21)

Parakiefferiella bathophila (K.) : (1) (4) (5) (9) (21)

* P. languida Br. : (2)

Parametriocnemus stylatus (K.) : (1) (4) (5) (21)

Paraphaenocladius impensus (Walk.) : (4) (5) (19)

$\rightarrow$ P. irritus (walk.) : (2)

$\rightarrow$ Paratrichocladius rufiventris (Mg.) : (1) (2) (4) (5) (21)

$\rightarrow$ P. skirwithensis (Edw.) : (1) (20)

$\rightarrow$ Paratrissocladius excerptus (walk.) (= Trissocladius fluviatilis $G.):(1) \cdot(5)$

- Psectrocladius limbatellus (Holm.) : (4)

$\rightarrow$ Pseudorthocladius curtistylus (G.) : (1) (5)

- Pseudosmittia holsata Th. Str. : (4) (5)

* $\rightarrow$ Ps. recta (Edw.) : (4) (5) 
Rheocricotopus atripes (K.) (= foveatus (Edw.)) : (2) (4)

Rh. chalybdeatus (Edw.) : (1) (2) (4) (5) (7) (21)

Rh. effusus (walk.) : (2) (4) (5) (21)

Rh. fuscipes (K.) : (1) (5) (7) (21)

* Rh. gallicus Lehm. : (8)

- Rh. glabricollis (Mg.) : (18)

Smittia aterrima (Mg.) : (18) (?21) (21)

$\rightarrow$ S. pratorum G. : (4) (21)

* Symposiocladius lignicola (K.) : (20, sub Orthocladius $\left.\ell . \mathrm{K}_{.}\right)$

Synorthocladius semivirens (K.) : (1) (2) (4) (5) (7) (9) (21)

Thienemannia gracei (Edw.) (= Metriocnemus gracei auct.) : (5)

Thienemanniella clavicornis $\mathrm{k}$. : (5)

$\rightarrow$ Th. obscura Br. : (?4) (21)

* Th. partita schlee : (?2)

$\rightarrow$ Th. vittata (Edw.) : (4) (5) (9)

Tuetenia bavarica (G.) : (?르)

T. calvescens (Edw.) : (1) (2) (4) (5) (7) (9) (21)

$\rightarrow T$. discoloripes (G.) : (1) (4, sub Eukieboriella verralli (Edw.) (9, idem) (21, sub $T$. verralli (Edw.))

- T. verralli (Edw.) : (4, sub Eukiefferiella discoloripes (G.)) (5, idem) (21, sub $T$. discoloripes (G.) )

Chironominae Chironomini (65 espèces)

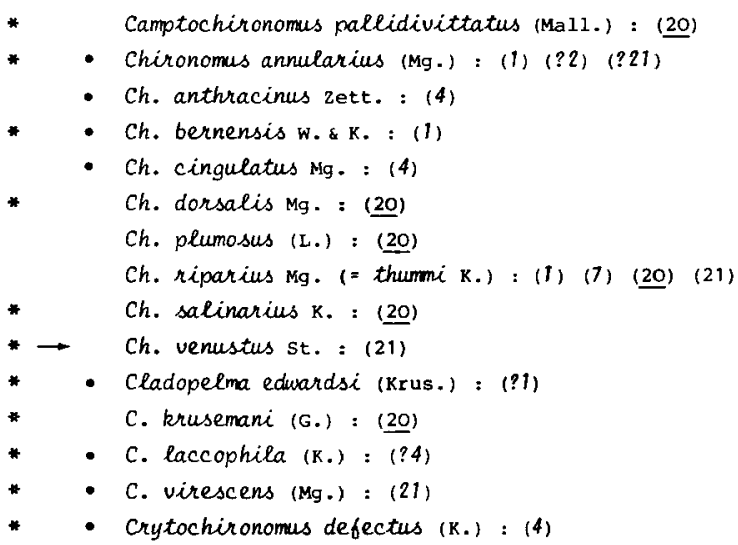


- C. rostratus $\mathrm{k}$. : (1) (7) (21)

C. supplicans (Mg.) : (1) (7) (? 20) (21)

$\rightarrow$ Cryptotendipes nigronitens (Edw.) : (?1) (?21, sub C. anomalus (K.))

- Demicryptochironomus vulneratus (zett.) : (1) (2) (21)

Dicrotendipes nervosus st. : (1) (2) (4) (5) (20) (21)

D. notatus (Mg.) : (1) (4) (7) (21)

Dolichopelma pusilla K. : (20)

Einfeldia dilatata (G.) : (20)

- Endochironomus albipennis (Mg.) : (1) (21)

E. dispar (Mg.) : (20)

E. impar (Walk.) : (20)

$E$. tendens (Fabr.) : (1) (4) (20)

Geyptotendipes gripekoveni $(\mathrm{K})=.(\underline{20})$

- G. pallens (Mg.) : (1) (21)

- G. paripes Edw. : (21)

- Harnischia curtilamellata (Mall.) : (?7) (?21)

- H. fuscimanus K. : (2) (?7) (?21)

- Kiefferulus tendipediformis (G.) : (1) (21)

- Microchironomus tener (K.) : (?1) (4)

Microtendipes chloris (Mg.) $(=$ pedellus (d. Geer) $)$ (2) (?4) (20)

* $\rightarrow$ M. confinis (Mg.) : (5)

M. rydalensis (Edw.) : (1) (ㅁ) ( 21)

* $\rightarrow$ Omisus caledonicus (Edw.) : (20)

- Parachironomus arcuatus (G.) : (1) (?4) (7) (21)

* $\rightarrow$ P. atriforceps (G.) : (20)

- P. frequens (Joh.) : (1) (21)

- P. vitiosus (G.) : (4)

Paracladopelma camptolabis (K.) : (?4) (20) (21)

- P. laminata (k.) : (2)

- Paralauterborniella nigrohalteralis (Ma11.) : (21)

Paratendipes albimanus (Mg.) : (1) (?21)

- Phaenopsectra albiventris (K.) : (1) (4)

Ph. flavipes (Mg.) : (1) (20) (21)

Polypedilum albicorne (Mg.) : (1) (2) (20) (21)

P. apfelbecki (Strobl.) : (4) (5) (20) (?21)

P. convictum (walk.) : (1) (4) (5) (9) (21)

P. cultellatum G. : (1) (21)

P. laetum (Mg.) : (7) () 
P. nubeculosum (Mg.) : (1) (4) (5) (20) (21)

$\rightarrow$ P. nubens (Edw.) : (?5) (21)

P. pedestre (Mg.) : (1) (2) (7) (20) (21)

- P. pullum (zett.) : (7)

P. quadriguttatum K. (= scalaenum (Schr.)) : (1) (2) (ㅁ) (21)

$P$. sordens (V. d. W) : $(\underline{20})$

- P. tritum (Walk.) : (4)

* Stenochironomus fascipennis (zett.) : (20) (21)

Stictochironomus histrio (Fabr.) : (2) (20)

* S. maculipennis (Mg.) : (1)

* $\rightarrow$ S. pictulus (Mg.) : (4) (ㅁ) (20)

* Xenochironomes xenolabis (K.) : (1) (7) (21)

\section{Chironominae Tanytarsini (43 espèces)}

cladotanytarsus atridorsum $\left(K_{0}\right)$ : (4) (ㅁ)

C. mancus (walk.) : (20) (21)

* C. vanderuvelpi (Edw.) : (4) (5)

Micropsectra atrofasciata (R.) : (1) (2) (4) (5) (7) (20) (21)

M. bidentata G. : (2) (5) (20) (21)

M. fusca (Mg.) : (20)

* M. globulifera (G.) : (5)

M. junci (Mg.) : (2)

M. notescens (walk.) : (1) (5) (20, sub M. praecox (Mg.)) (?21)

$\rightarrow$ Neozavrelia fuldensis Fitt. : (1) (2) (4) (5) (9) (21)

- Paratanytarsus austriacus (K.) : (4)

- P. bituberculatus (Edw.) : (1)

P. confusus pal. : (1) (2) (4) (5) (7) (9) (21)

- P. lauterborni (K.) : (21)

* P. quintuplex K. : (?21)

* - P. tenellulus (G.) : (1)

Rheotanytarsus curtistylus (G.) : (1) (4) (5) (9) (21)

$\rightarrow$ R. distinctissimus $(\mathrm{Br}$.$) : (1) (4) (5) (?21)$

- R. muscicola K. : (1) (2) (?21)

$\rightarrow$ R. nigricauda Fitt. : (1) (4) (5)

R. pentapoda (K.) : (1) (5) (?21)

- R. photophilus (G.) : (1) (7) (21)

* $\rightarrow$ R. reissi Leh. : (4) (9) (21)

$\rightarrow$ R. ? ringei Leh. : (1) (5) (7) (21) 
- Stempellina bausei (K.) : (1)

* S. subglabripennis (Br.) : (1) Stempellinella brevis (Edw.) : (1) (5) (21)

- Tanytarsus bathophilus $\mathrm{K}$. : (4)

T. brundini lind. : (1) (4) (5) (21)

* - T. curticornis $\mathrm{K}$. : (1)

T. debilis (Mg,) (= samboni Edw.) : (20)

$T$. ejuncidus (Walk.) : (1) (2) (? 21)

T. eminulus (walk.) : (1) (4) (5) (?7)

- T. fimbriatus Reiss \& Fitt. : (1)

* $\rightarrow$ T. gracilentus (Holm.) : (5)

* T. gregarius $\mathrm{K}$. : (20)

$T$. heusdensis G. : (1) (21)

T. holochlorus Edw. : (20)

T. lestagei G. : (?5) (20) (21)

$T$. pallidicornis (walk.) : (1) (4) (21)

T. palmeni Lind. : (20)

Virgatonytarsus arduennensis (G.) : ()

V. triangularis (G.) : (1) (4) (5) (7)

de $500 \mathrm{~m}$, limite altitudinale inférieure de la zone 8 des moyennes montagnes : 3 sur la Dordogne, 10 sur le Lot et 6 sur l'Aveyron. Nous avons inclus dans ce bilan les données recueillies dans Ies 53 stations situées dans la zone de piémont et susceptibles de préciser la distribution altitudinale de plusieurs espèces.

3. Les rivières du Sud-Est, affluents et sousaffluents du Rhône, surtout dans la partie Sud du département de l'Ardèche, entre $65 \mathrm{~m}$ et $730 \mathrm{~m}$ d'altitude (Serra-Tosio 1964, 1966, 1968, 1970, 1971,
1972, 1983 et sous-presse). Dans cette partie du Massif Central, les recherches ont essentiellement porté sur les Chironomidae Diamesinae.

L'analyse d'un nombre important de travaux récents et même parfois inédits nous permet de présenter le catalogue actualisé des Chironomidés du Massif Central et des basses régions avoisinantes (Tableau I).

Le bilan faunistique établi par sous-familles ou tribus est récapitulé dans le tableau II.

Tableau II : Bilan faunistique des Chironomidés du Massif Central et des basses régions avoisinantes. Espèces nouvelles pour la Zone 8 de la Limnofauna (1978).

\begin{tabular}{|c|c|c|c|c|c|c|c|c|}
\hline \multirow[t]{2}{*}{ Espèces } & \multirow[t]{2}{*}{ Tanypodinae } & \multirow[t]{2}{*}{ Buchono. } & \multirow[t]{2}{*}{ Diamesinae } & \multirow[t]{2}{*}{ Prodiam. } & \multirow[t]{2}{*}{ Orthocl. } & \multicolumn{2}{|c|}{ Chironominae } & \multirow[t]{2}{*}{ Total } \\
\hline & & & & & & Chironomini & Tanytarsini & \\
\hline \multirow{4}{*}{$\begin{array}{l}\text { Massif } \\
\text { Central s.l. } \\
500 \mathrm{~m} \\
\text { seulement } \\
\text { Nouvelles } \\
\text { pour } \mathrm{Z}_{8}\end{array}$} & & & & & & & & \\
\hline & 24 & 1 & 16 & 1 & 111 & 65 & 43 & 261 \\
\hline & 6 & 0 & 3 & 0 & 23 & 27 & 13 & 72 \\
\hline & 2 & 1 & 0 & 0 & 40 & 10 & 7 & 60 \\
\hline
\end{tabular}


Tableau III : Comparaison des bilans faunistiques des Chironomidés des Pyrénées (Laville \& Vinçon 1986) et du Massif Central.

\begin{tabular}{|c|c|c|c|c|c|c|c|c|}
\hline Espèces & Tanypodinae & Buchono. & Diamesinae & Prodiam. & Orthocl. & \multicolumn{2}{|c|}{$\begin{array}{c}\text { Chironominae } \\
\text { Chironomini Tanytarsini }\end{array}$} & Total \\
\hline $\begin{array}{l}\text { Massif } \\
\text { Central }\end{array}$ & & . & & & & & & \\
\hline Total & 24 & 1 & 16 & 1 & 111 & 65 & 43 & 261 \\
\hline Non Pyr. & 7 & 1 & 3 & 0 & 23 & 35 & 13 & 82 \\
\hline Pyrénées & & & & & & & & \\
\hline Total & 27 & 0 & 24 & 3 & 140 & 43 & 51 & 288 \\
\hline Non M.C. & 10 & 0 & 11 & 2 & 55 & 13 & 21 & 112 \\
\hline M.C. + Pyr. & & & & & & & & \\
\hline Communes & 17 & 0 & 13 & 1 & 85 & 30 & 30 & 176 \\
\hline Total & 34 & 1 & 27 & 3 & 163 & 78 & 64 & 370 \\
\hline$\%$ & 9,2 & 0,2 & 7,3 & 0,8 & 44,1 & 21,1 & 17,3 & 100 \\
\hline
\end{tabular}

Sur les 261 espèces recensées, 201 proviennent d'altitudes supérieures à $500 \mathrm{~m}$ et peuvent, de ce fait, être considérées comme apppartenant à la zone 8 de la Limnofauna Europaea (Fittkau \& Reiss 1978). 60 espèces, dont 36 Chironominae, restent localisées dans les basses régions avoisinantes et intéressent donc la zone 13 de la Limnofauna Europaea.

On peut comparer ces résultats au récent bilan fau. nistique établi pour les Pyrénées par Laville \& Vinçon (1986) (Tableau III). Le nombre total d'espèces actuellement connues dans ces deux régions montagneuses est comparable : 261 pour le Massif Central, 288 pour les Pyrénées, avec quelque 176 espèces communes.

82 espèces, dont 35 Chironomini, sont seulement signalées du Massif Central et 112 espèces, dont 55 Orthocladiinae, sont uniquement connues de la chaîne pyrénéenne.

370 espèces au total sont actuellement répertoriées pour l'ensemble des deux massifs montagneux, ce qui laisse augurer une diversité spécifique bien plus grande pour la France lorsque les faunes chironomidiennes des quatre autres régions - Jura + Vosges, Alpes, Corse et Plaines occidentales - seront à leur tour inventoriées. Les Orthocladiinae $(44,1 \%)$ et les Chironominae $(38,4 \%)$ sont les sous-familles dominantes, les Tanypodinae $(9,1 \%)$ et les Diame. sinae $(7,2 \%)$ formant le reste.

Ce bilan actuel est très proche de celui de la faune d'Irlande avec 347 espèces (Murray \& Ashe 1984); il re présente $90,9 \%$ de celui de la faune de Roumanie avec 407 espèces (Cure 1985) et $\mathbf{8 2 , 6 \%}$ de celui de la faune de Grande Bretagne avec 448 espèces (Cranston 1976).
Travaux cités

1. - Brouquet-Laglaire (Y.) 1985. Etude des Chirononnidés (Diptera) de quelques rivières polluées du bassin de la Garonne: Touyre, Agout, Dadou, Thoré, Bas-Lot. Thèse 3e cycle, Ecologie, Toulouse III : $159 \mathrm{P}$.

2. - C.E.M.A.G.R.E.F. 1981. Etude hydrobiologique de la Dordogne. Groupement de Bordeaux, section Qualité des eaux, étude $n^{\circ} 3,265 p+72 p$.

3. - Commission d'Etudes du lac d'Eguzon 1956. Le lac d'Eguzon. Contribution à l'étude physique, chimique et biologique d'un lac de barrage artificiel. Ann. Station centrale d'Hydrobiol. appl, 6: 1-232.

Cranston (P.S.) 1976. Chironomidae. In " A checklist of British Insects (G.S. Kloet \& W.D. Hincks). 2nd edition (revised). Part 5. Diptera \& Siphonaptera. Handbk Ident. Br. Insects, 11 (5) : 12-21.

Cure (V.) 1985. Chironomidae (Diptera-Nematocera) aus Rumänien unter besonderer Berücksichtigung jener aus dem hydrographishen Einzugsgebiet der Donau. Arch. Hydrobiol. Suppl., 68:163-217.

Fitkau (E.J.) \& Reiss (F.) 1978. Chironomidae. in Illies J. éd. Limnofauna Europaea: 404-440. G. Fisher, Stuttgart,

4. - Laville (H.) 1979. Etude de la derive des exuvies nymphales de Chironomides au niveau du confluent Lot-Truyère. Anrls Limnol, 15 (2) : 155-180.

5. - Laville (H.) 1981. Récoltes d'exuvies nymphales de Chironomides (Diptera) dans le Haut-Lot, de la source (1 $295 \mathrm{~m})$ au confluent de la Truyère (223 m). Annis Limnol., 17 (3) : 255-289.

6. - Laville (H.) \& Viaud-Chauvet (M.) 1983. Description de la nymphe de Telopelopia fascigera (Vemeaux) (Diptera, Chironomidae). Annls Limnol., 19 (1) : 25-28.

7. - Laville (H.) \& Viaud-Chauvet (M.) 1985. Etude comparée de la structure des peuplemerts de Chironomidés dans cinq rivières du Massif Central : relation entre cette structure et la qualité des eaux. Verh. Intemat. Verein. Limnol, 22 : 2261-2269. Laville (H.) \& Vinçon (G.) 1986. Inventaire 1986 des Chironomidés (Diptera) connus des Pyrénées. Ann/s Limnol., 22 (3) 239-251.

8. - Lehmann (J.) 1969. Die europäischen Arten der Gattung Rheo cricotopus Thien. und Harn. und drei neue Artvertreter dieser Gattung aus der Orientalis (Diptera, Chironomidae). Arch. Hydrobiol., 66: 348-381.

Murray (D.A.) Ashe (P.) 1984 . An inventory of the Irish Chi. ronomidae (Diptera). Mem. Amer. ent. Soc., $34: 223-233$. 
9. - Petitjeas (F.) 1981. Etude de f'herbier à Ranunculus fluitans d'Argentat sur Dordogne. These 3e cycle, Hydrobiologie, Univ. P. Sabatier, Toulouse, $188 \mathrm{p}$.

10. - Serra-Tosio (B.) 1964. Quelques Diamesini (Diptera, Chironomidae) du Dauphiné el du Vivarais. Description de quatre especes nouvelles. Trav. Lab. Hydrobiol. Piscic. Univ. Gre. noble, $56: 29-52$.

11. - Serra-Tosio (B.) 1966. Nouvelles données concermant la répartition et l'ecologie de quelques Chironomides Diamesini dans le Sud-Est de la France. Gewäss. Abwäss., 41/42: 124-128.

12. - Serra-Tosio (B.) 1968 . Taxonomie phylogénétique des Diamesini : les genres Potthastio Kieffer, Sympotthastia Pagast, Parapot thastia n.g. et Lappodiamese n.g. (Diptera, Chironomidae). Trav. Lab. Hydrobiol. Piscic. Univ. Grenoble, 59-60: $117-164$.

13. - Serra-Tosio (B.) 1970. Les Diamesini du groupe dampfi. Description d'une especie nouvelle (Diptera, Chironomidae). Trav: Lab. Hydrobiol. Piscic. Univ. Grenoble, 61 : 107-146.

14. - Serra-Tosio (B.) 1971. Contribution à l'étude taxonomique, phylogé nétique, biogéographique et écologique des Diamesini (Diptera, Chironomidae) d'Europe. These Univ. Scient Med. Grenoble, T.I : 1-303 : T.II : 304-462.

15. - Serra-Tosio (B.) 1972. Ecologie et biogéographie des Diamesini d'Europe (Diptera, Chironomidae). Trav. Lab. Hydrobiol. Piscic. Univ. Grenoble, 63 (1973) : 5-175.
16. - Serra-Tosio (B.) 1983. Données biogeographiques nouvelles sur les Diamesinae de Montagnes d'Asie et d'Afrique (Diptera, Chironomidae). 108e Congres National des Sociétés Savantes, Grenoble, 1983. Sciences, fasc. II : 257-268.

17. - Serra-Tosio (B.) sous presse. Révision des espèces ouestpaléarctiques et néarctiques de Boreohepıagia (Diptera, Chironomidae) avec des clés pour les larves, les nymphes et les imagos. Spixiana.

18. - Sicart (M.) \& Sardou (R.) 1963. Observation sur le vol de Smittia aternma (Chironomidae : Orthocladiinae), Bull. Soc. Hist. nat. Toulouse, $98:$ 293-294.

19. - Vaillant (F.) 1955. Recherches sur la faune madicole de France, de Corse et d'Afrique du Nord. Thèse Fac. Sci. Univ. Paris, A.2744, $n^{\circ} 3617: 258$ p.

20. - Verneaux (J.) 1968. Contribution à l'étude des Chironortides (Diptères, Nématocères) de la région de Besse-en-Chandesse (Puy-de-Dòme). Annls Sin. Biol. Besse-en-Chandesse, 3: 117.146.

21. - Viaud.Chauvet (M.) 1982. Analyse des récoltes d'exuvies nymphales de Chironomides dans les rivières du bassin du Tarn. Thèse de $3 e$ cycle, Ecologie, Toulouse III : $320 \mathrm{p}$.

22. - Willassen (E.) 1982. Descriptions and redescriptions of female Diamesa Meigen. 1935 (Diptera : Chironomidae) ; with a comparative morphology, keys, phylogenetic and biogeographical notes. Dissert. Dept. of Systematics, Museum of Zoology. University of Bergen, Norway : $316 \mathrm{p}$. 